\title{
Non-contiguous finished genome sequence of Phocaeicola abscessus type strain $7401987^{\mathrm{T}}$
}

\author{
Véronique Roux ${ }^{1 *}$, Catherine Robert ${ }^{1}$ and Didier Raoult ${ }^{1}$. \\ ${ }^{1}$ Aix Marseille Université, Faculté de médecine, Aix-Marseille Université, Marseille cedex, \\ France \\ * Correspondence: Véronique Roux (veronique.roux@univ-amu.fr)
}

Keywords: Corynebacterium timonense, Actinobacteria

\begin{abstract}
Phocaeic ola abscessus strain $7401987^{\top}$ is the sole member of the genus Phocaeicola. This bacterium is Gram-negative, non-spore-forming, coccoid to rod-shaped and motile by lophotrichous flagella. It was isolated from a human brain abscess sample. In this work, we describe a set of features of this organism, tog ether with the complete genome sequence and annotation. The 2,530,616 bp long genome contains 2,090 protein-coding genes and 54 RNA genes, including 4 rRNA operons.
\end{abstract}

\section{Introduction}

Phocaeicola abscessus strain 7401987T(CSUR $\mathrm{P} 22^{\mathrm{T}}=$ DSM $21584^{\mathrm{T}}=$ CCUG $55929^{\mathrm{T}}$ ) is the type strain of $P$. abscessus. This bacterium was isolated from a brain abscess sample from a 76-year-old patient who underwent neurosurgical intervention after cancer of the face [1]. It is a Gramnegative strictly anaerobic coccoid to rod-shaped bacterium. Currently, the genus Phocaeicola contains only one species [2].

Here we present a summary classification and a set of features for $P$. abscessus, together with the description of the non-contiguous finished genomic sequencing and annotation.

\section{Classification and features}

The 16S rRNA gene sequence of $P$. abscessus strain 7401987T was compared with sequences deposited in the Genbank database, confirming the initial taxonomic classification. Figure 1 shows the phylogenetic neighborhood of $P$. abscessus in a $16 \mathrm{~S}$ rRNA based tree. The bacterium was characterized in 2007. It was isolated in the Timone Hospital microbiology laboratory (Table 1).

Cells are coccoid 0.3-0.6 $\mu \mathrm{m}$ wide and 0.4-0.9 $\mu \mathrm{m}$ long) to rod-shaped (0.4-1.7 $\mu \mathrm{m}$ wide and 1.2-6.5 $\mu \mathrm{m}$ long) and motile by flagella in a lophotrichous arrangement. Optimal growth of strain $7401987 \mathrm{~T}$ occurs at $37^{\circ} \mathrm{C}$ with range for growth between 30 and $37^{\circ} \mathrm{C}$. Surface colonies on chocolate agar after 7 days incubation at $37{ }^{\circ} \mathrm{C}$ under anaerobic conditions were white, circular, regular, smooth, shiny, convex and $1 \mathrm{~mm}$ in diameter. The isolate was asaccharolytic. Activities of acid phosphatase, naphthol-AS-BI-phosphohydrolase, $\quad \mathrm{N}$-acetyl- $\beta$ glucosaminidase, $\alpha$-fucosidase, $\alpha$-galactosidase, $\beta$ galactosidase, $\beta$-galactosidase 6 -phosphate, $\alpha$ glucosidase, $\mathrm{N}$-acetyl- $\beta$-glucosaminidase, alkaline phosphatase, leucyl glycine arylamidase and alanine arylamidase were detected. The fatty acid profile was characterized by the predominance of anteiso- $\mathrm{C}_{15: 0} \quad(28.2 \%), \mathrm{C}_{16: 0} \quad(18.0 \%), \quad$ iso- $\mathrm{C}_{15: 0}$ $(12.3 \%)$ and iso- $\mathrm{C}_{17: 0}$ 3-он $(11.7 \%)$. The size and ultrastructure of cells were determined by negative staining transmission electron microscopy. (Figure 2). Cells are coccoid (0.3-0.6 $\mu \mathrm{m}$ wide and 0.4-0.9 $\mu \mathrm{m}$ long) to rod-shaped (0.4-1.7 $\mu \mathrm{m}$ wide and 1.2-6.5 $\mu \mathrm{m}$ long).

\section{Genome sequencing and annotation Genome project history}

The organism was selected for sequencing on the basis of its phylogenetic position and 16S rRNA similarity to other members of the order Bacteroidales and is part of study of the new species characterized in our laboratory. A summary of the project information is shown in Table 2. The EMBL accession number is CAKQ01000000 and consists of 39 contigs ( $\geq 500 \mathrm{bp}$ ) and 9 scaffolds. Table 2 shows the project information and its association with MIGS version 2.0 compliance. 
Table 1. Classification and general features of Phocaeicola abscessus strain $7401987^{\top}$

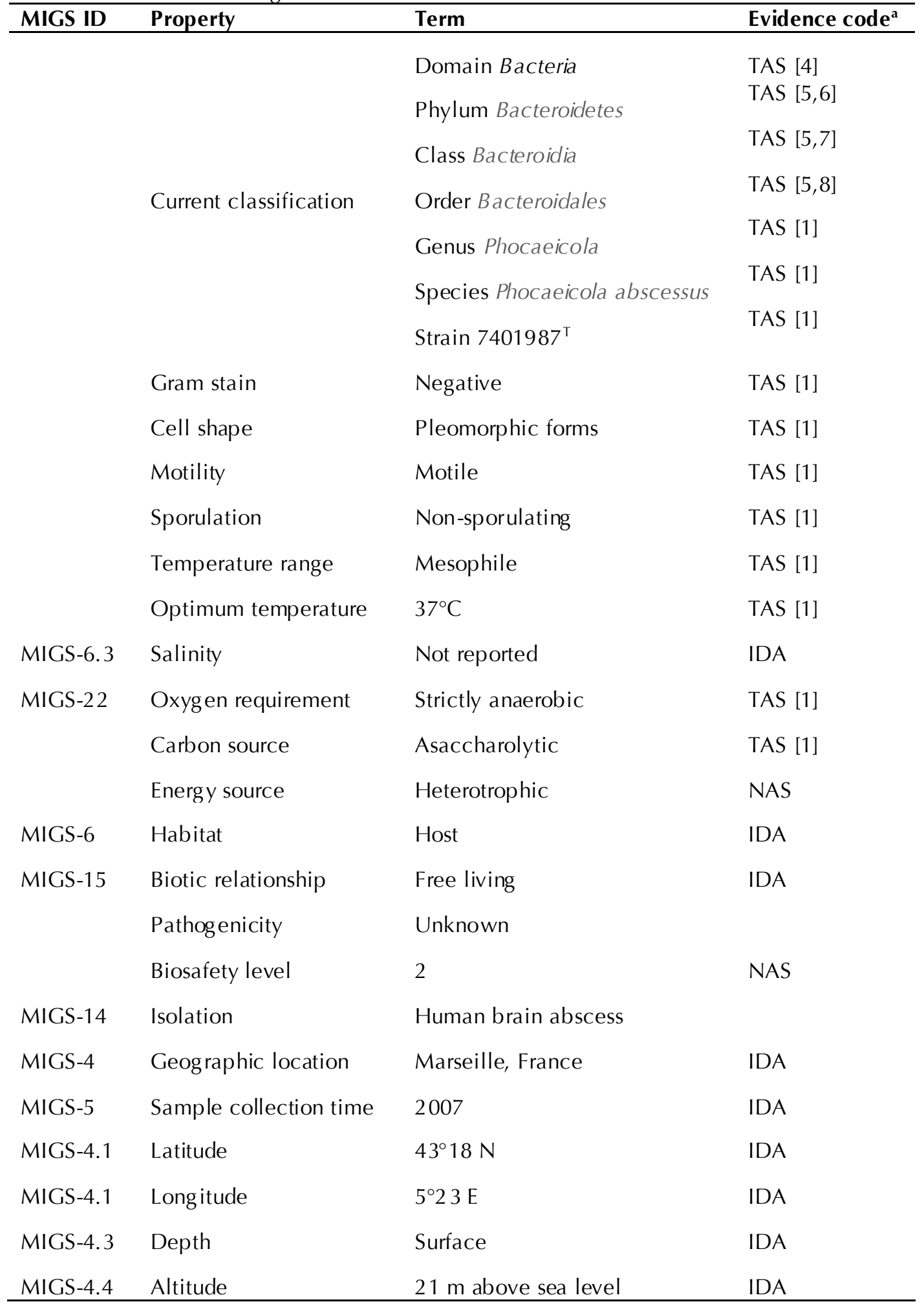

Evidence codes - IDA: Inferred from Direct Assay; TAS: Traceable Author Statement (i.e., a direct report exists in the literature); NAS: Non-traceable Author Statement (i.e., not directly observed for the living, isolated sample, but based on a generally accepted property for the species, or anecdotal evidence). These evidence codes are from the Gene Ontology project [9]. If the evidence is IDA, then the property was directly observed for a live isolate by one of the authors or an expert mentioned in the acknowledgements. 


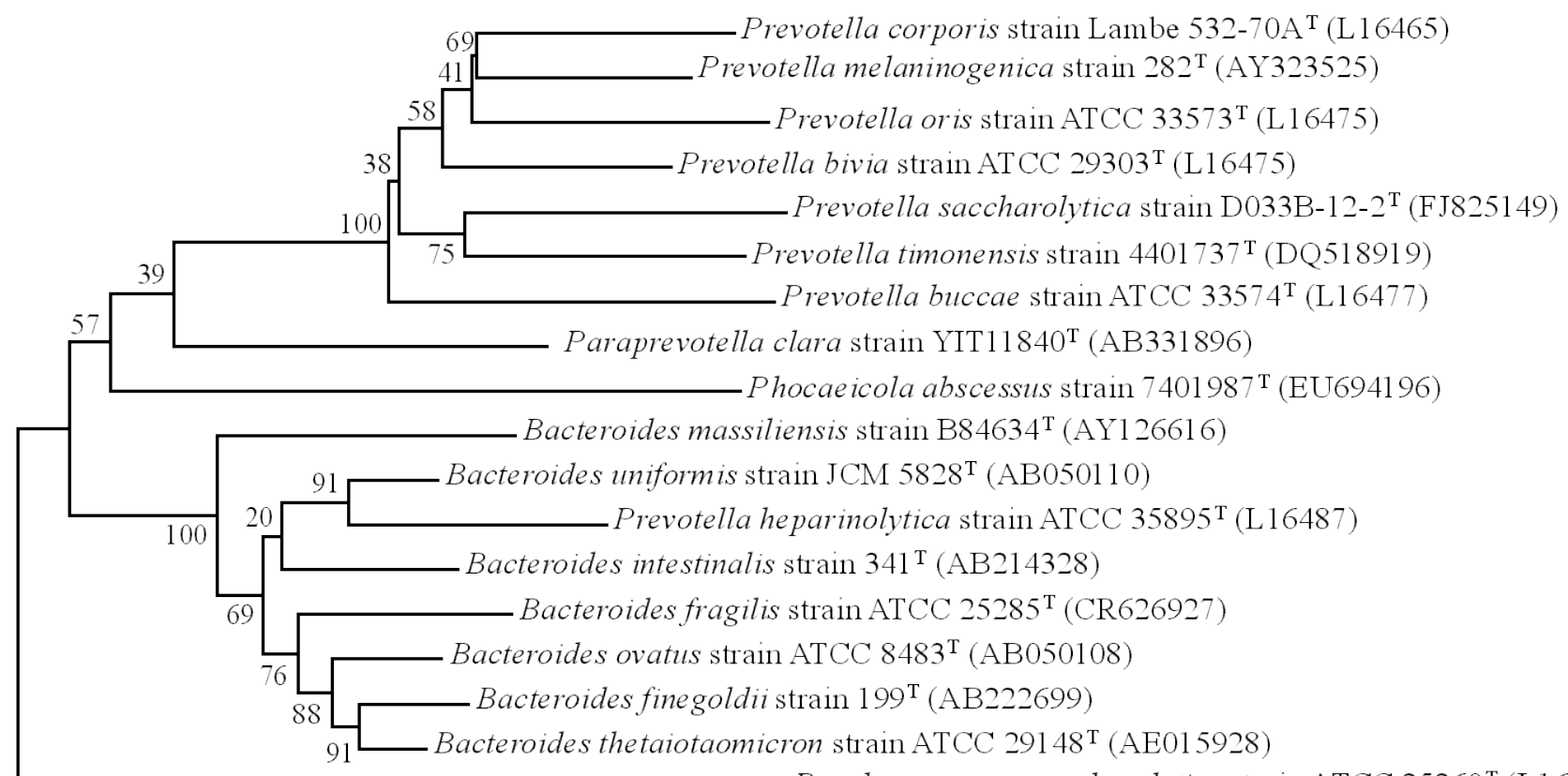

Porphyromonas asaccharolytica strain ATCC 25260 $(\mathrm{L} 16490)$

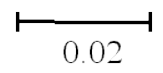

Figure 1. Phylogenetic tree hig hlighting the position of Phocaeicola abscessus strain 7401987 relative to bacteria included in the Prevotella, Bacteroides and Paraprevotella genera by comparison of 16S rRNA gene sequences. GenBank accession numbers are indicated in parentheses. Sequences were al igned using CLUSTALX, and phylogenetic inferences obtained using the neig hbor joining method within the MEGA 5 software [3]. Numbers at the nodes are percentages of bootstrap values obtained by repeating the analysis 1,000 times to generate a majority consensus tree. Porphyromonas asaccharolytica was used as outgroup. The scale bar represents 0.02 nucleotide change per nucleotide position.

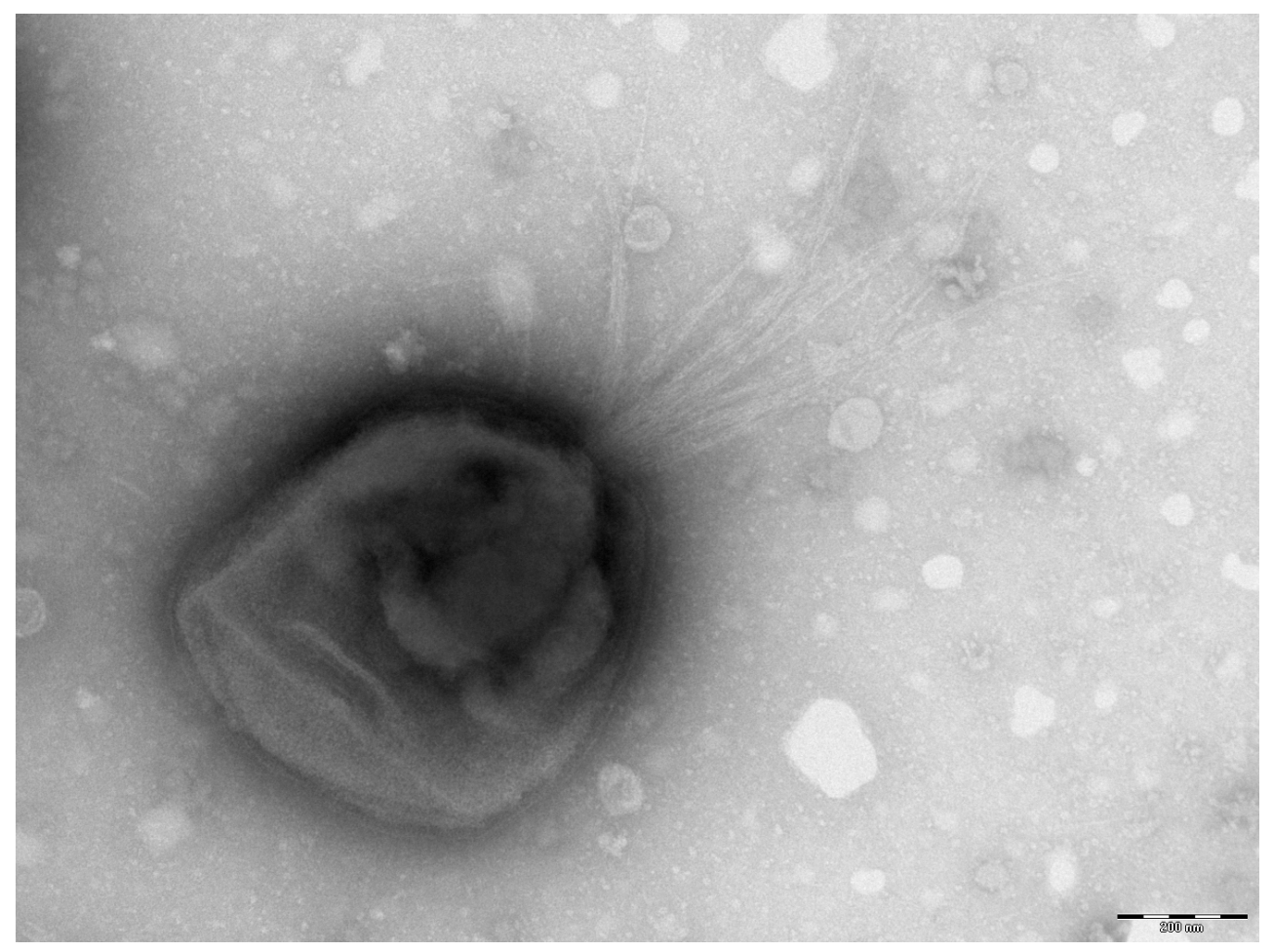

Figure 2. Transmission electron microscopy of $P$. abscessus strain 7401987, using a Morgani 268D (Philips) at an operating voltage of $60 \mathrm{kV}$. The scale bar represents $200 \mathrm{vm}$. 


\begin{tabular}{lll}
\multicolumn{2}{l}{ Table 2. Project } & information \\
\hline MIGS ID & Property & Term \\
\hline MIGS-31 & Finishing quality & High-quality draft \\
MIGS-28 & Libraries used & One paired end 3-kb library and one Shotg un library \\
MIGS-29 & Sequencing platforms & 454 GS FLX Titanium \\
MIGS-31.2 & Fold coverage & $35.9 \times$ \\
MIGS-30 & Assemblers & Newbler version 2.5.3 \\
MIGS-32 & Gene calling method & Prodigal \\
& EMBL ID & CAKQ01000000 \\
& EMBL Date of Release & February 12, 2012 \\
& Project relevance & Study of new species isolated in the URMITE \\
\hline
\end{tabular}

\section{Growth conditions and DNA isolation}

P. abscessus strain 7401987T, was grown anaerobically on chocolate agar at $37^{\circ} \mathrm{C}$. Ten petri dishes were spread and resuspended in $3 \mathrm{ml}$ of TE buffer. Three hundred $\mu \mathrm{l}$ of $10 \%$ SDS and $150 \mu \mathrm{l}$ of proteinase $\mathrm{K}$ were then added and incubation was performed overnight at $56^{\circ} \mathrm{C}$. The DNA was then extracted using the phenol/chloroform method. The yield and the concentration was measured by the Quant-it Picogreen kit (Invitrogen) on the Genios Tecan fluorometer at $88 \mathrm{ng} / \mu \mathrm{l}$.

\section{Genome sequencing and assembly}

Shotgun and 3-kb paired-end sequencing strategies were performed. The shotgun library was constructed with 500 ng of DNA with a GS Rapid library Prep kit (Roche). For the paired-end sequencing, $5 \mu \mathrm{g}$ of DNA was mechanically fragmented on a Hydroshear device (Digilab) with an enrichment size at 3-4 kb. The DNA fragmentation was visualized using a 2100 BioAnalyzer (Agilent) on a DNA labchip 7500 with an optimal size of 3.1 $\mathrm{kb}$. The library was constructed according to the 454 GS FLX Titanium paired-end protocol. Circularization and nebulization were performed and generated a pattern with an optimal size of 579 bp. After PCR amplification through 17 cycles followed by double size selection, the single stranded paired-end library was then quantified using a Genios fluorometer (Tecan) at $8,770 \mathrm{pg} / \mu \mathrm{L}$. The library concentration equivalence was calculated as $1.39 \mathrm{E}+10$ molecules/ $\mu \mathrm{L}$. The library was stored at $-20^{\circ} \mathrm{C}$ until further use.

The shotgun and paired-end libraries were clonally-amplified with $0.5 \mathrm{cpb}$ and $2 \mathrm{cpb}$ in 3 and $2 \mathrm{SV}$ emPCR reactions with the GS Titanium SV emPCR Kit (Lib-L) v2 (Roche). The yields of the emPCR were $9.63 \%$ and $10.3 \%$, respectively, in the 5 to $20 \%$ range from the Roche procedure. Approximately 790,000 beads for the shotgun application and for the $3 \mathrm{~kb}$ paired end were loaded on a GS Titanium PicoTiterPlate PTP Kit 70x75 and sequenced with a GS FLX Titanium Sequencing Kit XLR70 (Roche). The run was performed overnight and then analyzed on the cluster through the gsRunBrowser and Newbler assembler (Roche). A total of 311,276 passed filter wells were obtained and generated $35.9 \mathrm{Mb}$ with a length average of $282 \mathrm{bp}$. The passed filter sequences were assembled using Newbler with $90 \%$ identity and $40 \mathrm{bp}$ as overlap. The final assembly identified 9 scaffolds and 39 contigs ( $>500 \mathrm{bp}$ ).

\section{Genome annotation}

Open Reading Frames (ORFs) were predicted using Prodigal [10] with default parameters but the predicted ORFs were excluded if they were spanning a sequencing GAP region. The predicted bacterial protein sequences were searched against the GenBank database [11] and the Clusters of Orthologous Groups (COG) databases [12] using BLASTP. The tRNAscan-SE tool [13] was used to find tRNA genes, whereas ribosomal RNAs were found by using RNAmmer [14]. Transmembrane domains and signal peptides were predicted using TMHMM [15] and SignalP [16], respectively. ORFans of alignment length greater than 80 amino acids were identified if their BLASTp $E$-value was lower than 1e-03. If alignment lengths were smaller than 80 amino acids, we used an $E$-value of 1e-05. Such parameter thresholds have been used in previous works to define ORFans. 
To estimate the mean level of nucleotide sequence similarity at the genome level between $P$. abscessus and Prevotella timonensis, Bacteroides thetaiotaomicron and Paraprevotella clara, we compared the ORFs using only comparison sequences in the RAST server [17] at a query coverage of $\geq 70 \%$ and a minimum nucleotide length of $100 \mathrm{bp}$.

\section{Genome properties}

The genome is 2,530,616 bp long with a $47.31 \%$ GC content (Table 3, Figure 3). Of the 2,144 predicted genes, 2,090 were protein-coding genes, and 54 were RNAs. A total of 1,464 genes $(70.05 \%)$ were assigned a putative function. A total of 112 genes were identified as ORFans (5.39\%). The remaining genes were annotated as hypothetical proteins (436 genes (20.86\%)). The remaining genes were annotated as either hypothetical proteins or proteins of unknown functions. The distribution of genes into COGs functional categories is presented in Table 4. The properties and the statistics of the genome are summarized in Tables 3 and 4. Two CRISPRs were found using CRISPERfinder program online [18]. The first one on contig 1 includes at least 3 predicted spacer regions and the second one on contig 18 includes at least 53 predicted spacer regions.

Table 3. Nucleotide content and gene count levels of the genome

\begin{tabular}{|c|c|c|}
\hline Attribute & Value & $\%$ of total ${ }^{a}$ \\
\hline Genome size (bp) & $2,530,616$ & 100 \\
\hline DNA coding region (bp) & $2,284,358$ & 90.26 \\
\hline DNA G+C content $(b p)$ & $1,197,056$ & 47.31 \\
\hline Total genes & 2,144 & 100 \\
\hline RNA genes & 54 & 2.52 \\
\hline Protein-coding genes & 2,090 & 97.48 \\
\hline Genes with function prediction & 1,464 & 70.05 \\
\hline Genes assigned to COGs & 1,433 & 68.56 \\
\hline Genes with peptide sig nals & 554 & 26.51 \\
\hline Genes with transmembrane helices & 382 & 18.28 \\
\hline
\end{tabular}

a) The total is based on either the size of the genome in base pairs or the total number of protein coding genes in the annotated genome. 


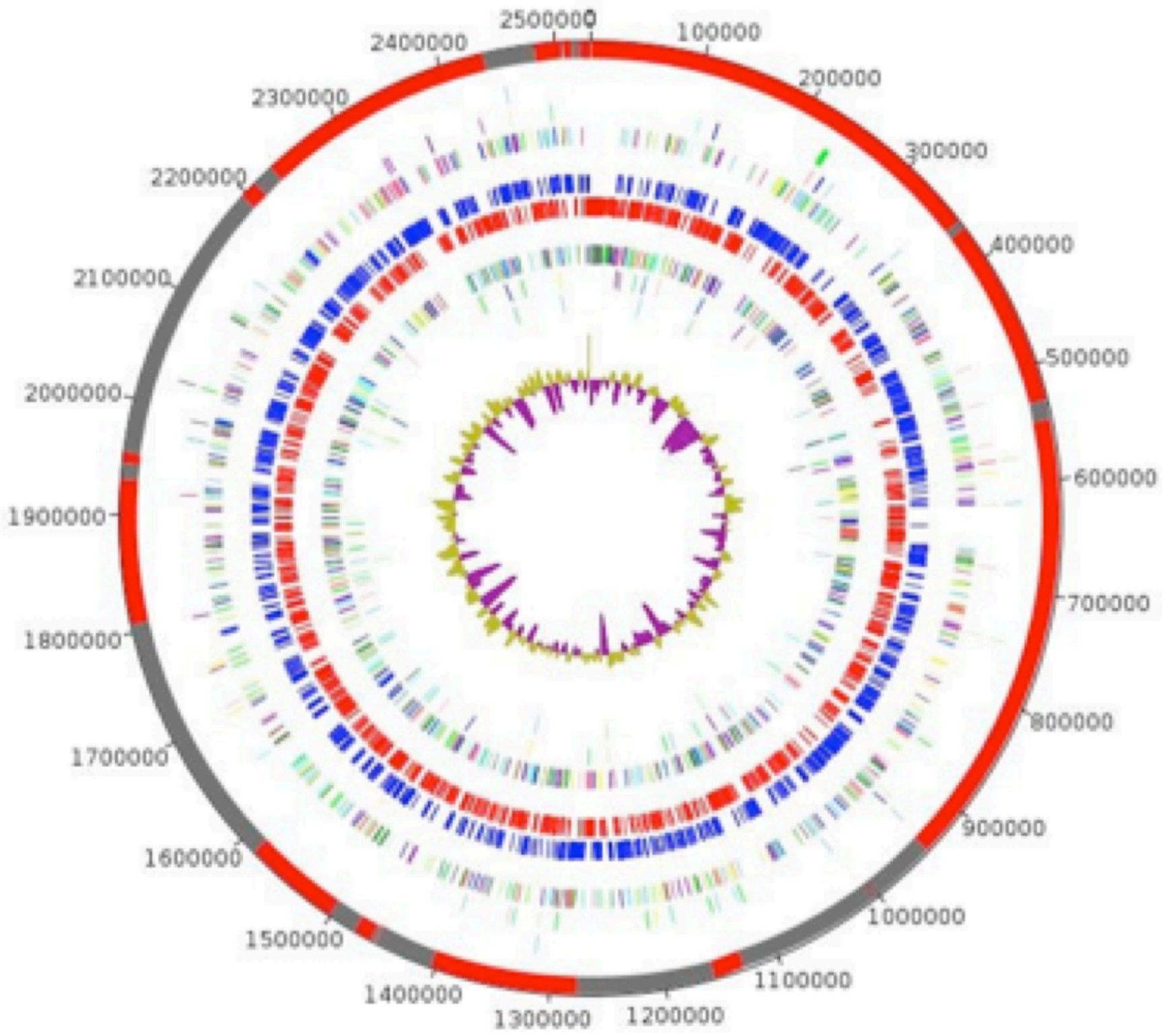

Figure 3. Graphical circular map of Phocaeicola abscessus genome. From outside to the center: Genes on the forward strand colored by COG categ ories (only genes assig ned to COG), genes on the reverse strand col ored by COG categ ories (only gene assig ned to COG), RNA genes (tRNAs green, rRNAs red), GC content and GC skew (three circles), GC content. 
Table 4. Number of genes associated with the 25 general COG functional categ ories

\begin{tabular}{crrl}
\hline Code & Value & \%age & Description \\
\hline J & 141 & 6.75 & Translation \\
A & 0 & 0 & RNA processing and modification \\
K & 82 & 3.92 & Transcription \\
L & 103 & 4.93 & Replication, recombination and repair \\
B & 0 & 0 & Chromatin structure and dynamics \\
D & 20 & 0.96 & Cell cycle control, mitosis and meiosis \\
Y & 0 & 0 & Nuclear structure \\
V & 36 & 1.72 & Defense mechanisms \\
T & 50 & 2.39 & Signal transduction mechanisms \\
M & 143 & 6.84 & Cell wall/membrane biog enesis \\
N & 2 & 0.10 & Cell motility \\
Z & 1 & 0.05 & Cytoskeleton \\
W & 0 & 0 & Extracellular structures \\
U & 29 & 1.39 & Intracellular trafficking and secretion \\
O & 63 & 3.01 & Posttranslational modification, protein turnover, chaperones \\
C & 86 & 4.11 & Energy production and conversion \\
G & 119 & 5.69 & Carbohydrate transport and metabolism \\
E & 122 & 5.84 & Amino acid transport and metabolism \\
F & 52 & 2.49 & Nucleotide transport and metabolism \\
H & 81 & 3.88 & Coenzyme transport and metabolism \\
I & 47 & 2.25 & Lipid transport and metabolism \\
P & 89 & 4.26 & Inorganic ion transport and metabolism \\
Q & 20 & 0.96 & Secondary metabolites biosynthesis, transport and catabolism \\
R & 225 & 10.77 & General function prediction only \\
S & 78 & 3.73 & Function unknown \\
\hline & 657 & 31.44 & Not in COGs \\
\hline
\end{tabular}

The total is based on the total number of protein coding genes in the annotated genome.

\section{Comparison with other genomes}

Phocaeicola abscessus is the sole bacterium included in the genus Phocaeicola. We compared the genome of $P$. abscessus with those of Prevotella timonensis (CBQQ010000001) Paraprevotella clara (AFFY01000000) and Bacteroides thetaiotaomicron (AE015928.1). P. abscessus showed a mean nucleotide sequence similarity of $76.40 \%, 77.06 \%$ and $77.52 \%$ at the genome level (range 70-92.25\%, 70.04-95.51\% and 70.04-
93.02\%) with P. timonensis, P. clara and B. thetaiotaomicron, respectively. Presently, the family to which $P$. abscessus belongs is undetermined and the sole comparison based on nucleotide sequence similarity may not be sufficient to answer this question. In the future, further comparison of the genomes will allow us to find traits to classify the genus Phocaeicola in one of these 3 families or to create a new family, the family Phocaeicolaceae. 


\section{Acknowledgements}

The authors thank Mr. Julien Paganini at Xegen Company (www.xegen.fr) for automating the genomic annotation process and Laetitia Pizzo for her technical assistance.

\section{References}

1. Al Masalma M, Raoult D, Roux V. Phocaeicola abscessus gen. nov., sp. nov., an anaerobic bacterium isolated from a human brain abscess sample. Int J Syst Evol Microbiol 2009; 59:2232-2237. PubMed http://dx.doi.org/10.1099/ijs.0.007823-0

2. Euzéby JP. List of Bacterial Names with Standing in Nomenclature: a folder available on the Internet. Int J Syst Bacteriol 1997; 47:590-592. PubMed http://dx.doi.org/10.1099/00207713-47-2$\underline{590}$

3. Tamura K, Peterson D, Peterson N, Stecher G, Nei M, Kumar S. MEGA5: Molecular Evolutionary Genetics Analysis using Maximum Likelihood, Evolutionary Distance, and Maximum Parsimony Methods. Mol Biol Evol 2011; 28:2731-2739. $\underline{\text { PubMed }}$ http://dx.doi.org/10.1093/molbev/msr121

4. Woese CR, Kandler O, Wheelis ML. Towards a natural system of organisms: proposal for the domains Archaea, Bacteria, and Eucarya. Proc Natl Acad Sci USA 1990; 87:4576-4579. PubMed http://dx.doi.org/10.1073/pnas.87.12.4576

5. Validation List No. 143. Int J Syst Evol Microbiol 2012; 62:1-4.

6. Krieg NR, Ludwig W, Euzéby J, Whitman WB. Phylum XIV. Bacteroidetes phyl. nov. In: Krieg NR, Staley JT, Brown DR, Hedlund BP, Paster BJ, Ward NL, Ludwig W, Whitman WB (eds), Bergey's Manual of Systematic Bacteriology, Second Edition, Volume 4, Spring er, New York, 2011, p. 25.

7. Krieg NR. Class I. Bacteroidia class. nov. In: Krieg NR, Staley JT, Brown DR, Hedlund BP, Paster BJ, Ward NL, Ludwig W, Whitman WB (eds), Bergey's Manual of Systematic Bacteriology, Second Edition, Volume 4, Springer, New York, 2011, p. 25.

8. Krieg NR. Order I. Bacteroidales ord. nov. In: Krieg NR, Staley JT, Brown DR, Hedlund BP, Paster BJ, Ward NL, Ludwig W, Whitman WB (eds), Bergey's Manual of Systematic Bacteriology, Second Edition, Volume 4, Springer, New York, 2011, p. 25.

9. Ashburner M, Ball CA, Blake JA, Botstein D, Butler $\mathrm{H}$, Cherry JM, Davis AP, Dolinski K, Dwight SS, Eppig JT, et al. Gene ontology: tool for the unification of biology. The Gene Ontology Consortium. Nat Genet 2000; 25:25-29. PubMed http://dx.doi.org/10.1038/75556

10. Prodigal http://prodigal.ornl.gov/

11. GenBank database. http://www.ncbi.nlm.nih.gov/genbank

12. Tatusov RL, Galperin MY, Natale DA, Koonin EV. The COG database: a tool for genome-scale analysis of protein functions and evolution. Nucle ic Acids Res 2000; 28:33-36. PubMed http://dx.doi.org/10.1093/nar/28.1.33

13. Lowe TM, Eddy SR. tRNAscan-SE: a prog ram for improved detection of transfer RNA genes in genomic sequence. Nucleic Acids Res 1997; 25:955-964. PubMed

14. Lagesen K, Hallin P, Rodland EA, Staerfeldt $\mathrm{HH}$, Rognes T, Ussery DW. RNAmmer: consistent and rapid annotation of ribosomal RNA genes. Nucleic Acids Res 2007; 35:3100-3108. PubMed http://dx.doi.org/10.1093/nar/gkm160

15. Krogh A, Larsson B, von Heijni G, Sonnhammer EL. Predicting transmembrane protein topology with a hidden Markov model: application to complete genomes. J Mol Biol 2001; 305:567580. $\underline{\text { PubMed }}$ http://dx.doi.org/10.1006/jmbi.2000.4315

16. Bendtsen JD, Nielsen H, von Heijne G, Brunak S. Improved prediction of signal peptides: Sig nalP 3. 0. J Mol Biol 2004; 340:783-795. PubMed http://dx.doi.org/10.1016/j.jmb.2004.05.028

17. Aziz RK, Bartels D, Best AA, DeJong h M, Disz T, Edwards RA, Formsma K, Gerdes S, Glass EM, Kubal M, et al. The RAST Server: Rapid Annotations using Subsystems Technology. BMC Genomics 2008; 9:75-89. PubMed http://dx.doi.org/10.1186/1471-2164-9-75

18. http://crispr.u-psud.fr/Server/ 